\title{
SOLVABLE GROUPS HAVING SYSTEM NORMALIZERS OF PRIME ORDER
}

\author{
BY \\ GARY M. SEITZ
}

\begin{abstract}
Let $G$ be a solvable group having system normalizer $D$ of prime order. If $G$ has all Sylow groups abelian then we prove that $l(G)=l\left(C_{G}(D)\right)+2$, provided $l(G) \geq 3$ (here $l(H)$ denotes the nilpotent length of the solvable group $H$ ). We conjecture that the above result is true without the condition on abelian Sylow subgroups. Other special cases of the conjecture are handled.
\end{abstract}

Let $H$ be a finite solvable group and $A$ a group of automorphisms of $H$. There are many results that give bounds for the nilpotent length of $H$ in terms of certain properties of $A$. In this note we are concerned with the case where $A$ has prime order and $A$ is a system normalizer of the group $G=H A$. That is, we assume that $G$ has no central chief factor contained in $H$. In this situation there is surprisingly exact information available. We conjecture that $l(G)=l\left(C_{G}(A)\right)+2$ provided $l(G) \geq 3$ (by $l(K)$ we mean the nilpotent length of the solvable group $K$ ). In this paper we prove special cases of the conjecture. For example if $G$ is an $A$-group (all Sylow subgroups abelian) or if $l(G)=4$, then the conjecture is true. We remark that these results are best possible in the sense that if either condition $|A|$ prime or $A$ a system normalizer of $G=H A$ is relaxed then examples can be constructed showing that precise equalities on $l(G)$ in terms of $l\left(C_{G}(A)\right)$ cannot always be expected.

We call attention to the preliminary Propositions 2 and 3, where we prove results that guarantee the existence of fixed points contained in fixed cosets, in the case of nonrelatively prime action.

Throughout the paper all groups will be solvable. If $G$ is a group, $l(G)$ is the nilpotent length of $G$. We let $\pi^{i}$ denote the saturated formation of groups of nilpotent length at most $i$.

The following results can be used to obtain fixed points in fixed cosets, in the case of nonrelatively prime action. We reduce the situation to Theorem 3.3 of [2].

Proposition 1. Let $P$ be a cyclic p-group acting fixed-point-free on a group Q. Let $P Q$ act on a p-group $R$ so that $Q$ acts on $R$ without fixed points. If $H / K$

Received by the editors August 16, 1972.

AMS (MOS) subject classifications (1970). Primary 20D10, $20 \mathrm{D} 45$.

Key words and phrases. Nilpotent length, system normalizers.

Copyright (c) 1973, American Mathematical Society 
is $P Q R$-invariant and $H \leq R$, then $C_{H / K}(P)=C_{H}(P) K / K$.

Proof. Suppose $x K \in C_{H / K}(P)$. Then $P^{x} K=P K$ and $K Q P=K Q P^{x}$. Also the chief factors of $K Q P$ contained in $K Q$ are eccentric, so $P$ is a system normalizer of $K Q P$. Theorem 3.3 of [2] implies that $P, P^{x}$ are conjugate in $K Q P$. Thus there exist elements $k \in K, q \in Q$ such that $P^{x}=P^{k q}$. Then $P H / H=$ $P^{x} H / H=P^{k q} H / H=P^{q} H / H$ and it follows that $q=1$. Thus $P^{x}=P^{k}$ and $x k^{-1} \epsilon$ $N(P) \cap H \leq C(P)$. This completes the proof of Proposition 1.

Corollary 2. Let $l(G)=3$ and suppose that a system normalizer $D$ of $G$ is cyclic of prime order. If $H / K$ is a $G$-invariant factor of $G^{\pi^{2}}$, then each coset of $K$ in $H$ centralized by $D$ contains an element centralized by $D$.

Proof. Let $|D|=p$. Since $G^{r^{2}}$ is nilpotent, $H / K$ is nilpotent and we may assume that $H / K$ is a $p$-group. Also we may assume that $K$ is a $p$-group. Let $L$ be an $\Re^{2}$-normalizer of $G$ with $D \leq L\left[1\right.$, Theorem 4.9]. Then $L=D L_{0}$ where $L_{0}=L \cap G^{r}$. Since $H$ is hypereccentric and $H$ is a $p$-group, the $p$-complement $Q$ of $L_{0}$ acts without fixed points on $H$. As $D$ acts fixed-point-freely on $Q$, we can apply Proposition 1.

The following result is similar in nature to Proposition 1. We will not need this result in what follows although it can be used in similar situations. We remark that an alternate proof is possible using a result of Thompson (Theorem 1 of [3]).

Proposition 3. Let $P$ be a cyclic p-group, $p>2$, and suppose $P$ acts fixedpoint-freely on a group $Q$. Let $P Q$ act on the class 2 p-group $R$, and assume $[R, Q]=R$. If $H$ is a $P Q$-invariant subgroup of $Z(R)$ such that $R / H$ is elementary abelian, then each coset of $H$ in $R$ fixed by $P$ contains an element fixed by $P$.

Proof. Let $G=R Q P$ be a minimal counterexample. Suppose that $P$ fixes the coset $x H$. Write $H=H_{1} \times H_{2}$ where $H_{1}=C_{H}(Q)$ and $H_{2}=[H, Q]$, and suppose $H_{1}>1$ and $H_{2}>1$. Then from the groups $G / H_{1}$ and $G / H_{2}$ we find elements $x_{1}, x_{2}$ in $x H$ such that $P$ fixes $x_{1} H_{1}$ and $x_{2} H_{2}$. Consequently there exist elements $b_{1} \in H_{1}, b_{2} \in H_{2}$ such that $P$ fixes $x_{1} H_{1}=x b_{2} H_{1}$ and $x_{2} H_{2}=x b_{1} H_{2}$. Let $P=$ $\langle g\rangle$. There exist elements $t_{1} \in H_{1}, t_{2} \in H_{2}$ such that $\left(x b_{2}\right)^{g}=x b_{2} t_{1}$ and $\left(x b_{1}\right)^{g}$ $=x b_{1} t_{2}$. But $x b_{1}=x b_{2}\left(b_{2}^{-1} b_{1}\right)$, and so $\left(x b_{1}\right)^{8}=x b_{2} t_{1}\left(b_{2}^{-1}\right)^{8} b_{1}^{g}=x b_{1} t_{2}$. It follows that $b_{2}\left(b_{2}^{-1}\right)^{g} t_{2}^{-1}=b_{1}\left(b_{1}^{-1}\right)^{g} t_{1}^{-1}$. Since $g \in N(Q), g$ normalizes $H_{1}$ and $H_{2}$, and consequently $t_{2}=b_{2}\left(b_{2}^{-1}\right)^{g}$ and $\left(x b_{1}\right)^{g}=x b_{1} b_{2}\left(b_{2}^{-1}\right)^{g}$. This gives $\left(x b_{1} b_{2}\right)^{g}=$ $x b_{1} b_{2}$. Therefore $H_{1}=1$ or $H_{2}=1$.

If $H_{1}=1$, then since $R / H$ is abelian and $[R, Q]=R$, we have $Q$ acting on $R$ without fixed points. In this case Proposition 1 gives a fixed point of $P$ in $x H$. 
Thus we have $H_{2}=1$ and $H \leq Z(R Q)$. We next show that $H=Z(R)$. If $H<Z(R)$ then write $Z(R)=H \times L$ where $L=[Z(R), Q]$. From the group $G / L$ we find an element $b \in H$ such that $x b L$ is fixed by $P$. Then $(x b)^{g}=x b l$ for some $l \in L$, and $x^{8}=x b_{1}$ for some $b_{1} \in H$. It follows that $l=1$ and $g$ fixes $x h$. Thus $H=$ $Z(R)$ as claimed. Suppose $[H, P] \neq 1$. Then considering the series $H>[H, P]>$ $[H, P, P]>\cdots>1$ we find an element of the form $1 \neq z=\left[g, z_{1}\right]$ where $z$ is centralized by $P$ and $z_{1} \in H$. Then $\langle z\rangle \leq Z(G)$ and from $G /\langle z\rangle$ we have an element $b \in H$ such that $P$ fixes $x b\langle z\rangle$. Then $(x b)^{g}=x b z^{i}$ for some $i,(x b)^{g}=x b\left[g, z_{1}^{i}\right]$, and $\left(x b z_{1}^{i}\right)^{g}=x b z_{1}^{i}$. Therefore we must have $H=Z(G)$.

If $H=Z(R)$ is not cyclic then letting $N_{1}, N_{2}$ be proper subgroups of $Z(R)$ with $N_{1} \cap N_{2}=1$ we get a contradiction by considering $G / N_{1}$ and $G / N_{2}$. Therefore $Z(R)$ is cyclic and since $[Q, R]=R, R$ is extraspacial. Thus, $\left|R: \Omega_{1}(R)\right| \leq p$ and hence $R=\Omega_{1}(R)$. Now let $A$ be the subgroup of the automorphism group of $R$ that centralizes $Z(R)$. Then $P Q \leq A$ and $A$ is the semidirect product of $\operatorname{Inn}(R)$ with $L \cong \mathrm{Sp}(V)$, where $V$ is the vector space $R / Z(R)$. This may be checked as follows. First show that $\operatorname{Inn}(R)$ is the group of automorphisms of $R$ centralizing $R / Z(R)$. Then for each $g \in \mathrm{Sp}(V)$ it is possible to produce an automorphism of $R$ having the appropriate action on $R / Z(R)$ and centralizing $R$. This proves that $A / \operatorname{Inn}(R)$ $\cong \mathrm{Sp}(V)$ and the only question is whether the extension splits. Since $p$ is odd, $\mathrm{Sp}(V)$ has a central involution, and consequently $A$ contains an involution $t$ such that $t$ inverts $\operatorname{Inn}(R)$. Then $L=C_{A}(t) \cong \mathrm{Sp}(V)$.

Now $\operatorname{Inn}(R)$ is $L$-isomorphic to $V$. Since $(|Q|, p)=1$ we conjugate if necessary to assure $Q \leq L$. $[Q, R]=R$ implies that $[Q$, Inn $R]=\operatorname{Inn} R$ and it follows that $N_{A}(Q) \leq L$. In particular $P Q \leq L$ and $P Q$ centralizes the central involution $t$ of $L$. Now $\langle x, Z(R)\rangle=Z(R) \times R_{0}$ where $t$ centralizes $Z(R)$ and inverts $R_{0}$. $P$ centralizes $t$ and hence normalizes $R_{0}$. However $P$ centralizes $\langle x, Z(R)\rangle / Z(R)$ and $R_{0}$ contains precisely one element of $x Z(R)$. Therefore $P$ centralizes $R_{0}$ and an element in $x Z(R)$. This completes the proof of Proposition 3.

The next lemma is trivial but useful.

Lemma 4. Let $l(G)=n$ and $H \leq G$. If $H$ bas a chief factor $M / N$ such that $l\left(H / C_{H}(M / N)\right)=n-1$, then $l(H)=n$.

Proof. $l(H) \leq n$ and Fit $(H)$ centralizes each chief factor of $H$.

Theorem 5. Let $G$ be a finite solvable group baving system normalizer $D$ of prime order. If $l(G)=4$, then $l\left(C_{G}(D)\right)=2$.

Proof. Suppose the result false and let $G$ be a counterexample of minimal order. Write $G=D G^{\pi}$, where $G^{r}$ is the nilpotent residual of $G$ and where $|D|=p$. Then $D \cap G^{\pi}=1, G^{\pi}$ is hypereccentric, and $C_{G}(D)=D \times C_{G} \pi(D)$. Also $D$ acts fixed-point-freely on $G^{\pi / G{ }^{2}}$, so that $C_{G} \pi^{(D)} \leq G^{\pi^{2}}$ and $l\left(C_{G}(D)\right)=$ 
$l\left(C_{G} \pi(D)\right) \leq l\left(G^{\pi^{2}}\right)=2$. As $G$ is a counterexample we have $C_{G} r^{(D)}$ nilpotent. Let $N$ be a minimal normal subgroup of $G,|N|=q^{a}$, $q$ prime.

We claim that $N=G^{r^{3}}=$ Fit $(G)$ and $N$ is the unique minimal normal subgroup of $G$. It suffices to prove the equalities, and we first show that $N=G r^{3}$. Suppose that $q \neq p$. Then $C_{G / N}(D N / N)=C(D) N / N$. Thus if $G^{\pi^{3}} \npreceq N$, then $C(D) N / N$ has length 2 , contradicting the choice of $G$. Thus $N=G^{r^{3}}$. Now suppose $q=p$. Since $D$ is fixed-point-free on $G \pi / G \pi^{2}, N \leq G^{\pi^{2}}$. Let $T$ be an $\pi^{3}$. normalizer of $G$ containing $D\left[1\right.$, Theorem 4.9] and consider the group $G_{0}=N T$. Since $G^{r^{3}}$ is nilpotent and $G=G^{r^{3}} T$, it follows that $N$ is a minimal normal subgroup of $G_{0}$ and by the covering properties of $T$, either $N \leq T$ or $N \cap T=1$. If $l\left(G_{0}\right)=4$, then since $D$ is a system normalizer of $G_{0}$, we conclude from the minimality of $G$ that $G=G_{0}$ and $N=G r^{3}$. Now suppose $l\left(G_{0}\right)=3$. Then $N \leq T$ and $G_{0}=T$ has nilpotent length 3. Also $l\left(G / C_{G}(N)\right)=2$. Indeed, $G=G^{{ }^{3}} T$ and both $G^{r^{3}} \leq \mathrm{Fit}(G)$ and Fit $(T)$ centralize $N$. This shows $l\left(G / C_{G}(N)\right) \leq 2$. If $l\left(G / C_{G}(N)\right)=1$, then $G^{\pi} \leq C_{G}(N)$, and since $N$ is a p-group, $N \leq Z(G)$, a contradiction. In particular $N \leq Z\left(G^{r^{2}}\right)$. Let $C=C_{G} r(D N / N)$ and suppose $G^{\pi^{3}} \leq N$. The minimality of $G$ implies $l(C / N)=2$. If $G^{r^{3}}$ is not a $p$-group, let $M$ be a minimal normal subgroup of $G, M \leq O_{p},\left(G^{r^{3}}\right)$. By the above it follows that $M=$ $G^{r^{3}}$. Since $l(C / N)=2$ and since $C \leq G^{r^{2}}$, some element $x N$ of $C / N$ of order prime to $|M|$ does not centralize $C_{M}(D)$. Otherwise $C_{M}(D) N / M$ would be hypercentral in $C / N$ and since $C / C_{M}(D) N$ is nilpotent, this would contradict $l(C / N)=2$. Since $G / N=(T / N)(M N / N)$ and since $(|x N|,|M|)=1$, we may assume that $x \in T$. Corollary 2 implies that there is a $y \in C(D)$ such that $y N=x N$. As $N \leq C(M)$, we have $C_{M}(D)\langle y\rangle$ nonnilpotent and contained in $C(D)$. Thus we may assume that $G \pi^{3}$ is a p-group. Let $g \in C \leq G \pi^{2}$ and $b \in D$. Then $C_{C}(b)^{8}=C_{C}\left(b^{8}\right)=C_{C}(b n)$ for some $n \in N$. Since $N \leq$ $Z\left(G^{r^{2}}\right)$, this gives $C_{C}\left(b^{8}\right)=C_{C}(b)$ and $C_{C}(D) \unlhd C$. Also all $p^{\prime}$-elements of $C$ are contained in $C_{C}(D)$. Indeed if $x$ is a $p^{\prime}$-element of $C$, then $x \in C(D N / N)$ and $x \in C(N)$ implies $x \in C(D N)$. Since $C(D)$ is nilpotent, $C$ contains a normal nilpotent $p$-complement. On the other hand $G^{r^{3}}$ is a $p$-group and $C \leq G r^{2}$. Thus $C$ also contains a normal Sylow $p$-group. This implies that $C$ is nilpotent, whereas $l(C / N)=2$. This contradiction shows that $N=G r^{3}$ in all cases. In particular $N$ is unique. With $T$ as above, we have Fit $(G)=N($ Fit $(G) \cap T)$. Since $N$ is minimal normal in $G$, Fit $(G) \cap T$ centralizes $N$ and Fit $(G) \cap T \unlhd G$. The uniqueness of $N$ shows that Fit $(G) \cap T=1$. This proves the claim.

We next claim that $G$ can be expressed $G=N R Q D$ where $D \leq N(Q), Q$ minimal normal in $Q D, Q D \leq N(R)$, and $R$ is a minimal normal subgroup of $R Q D$. To see this first write $G=N T$ where $T$ is an $\pi^{3}$-normalizer of $G$ and $D \leq T$. Let $L$ be an $\Re^{2}$-normalizer of $T$ with $D \leq L$. Then $L=D W$ with $W=L \cap G^{\bar{r}}$. Let $1 \neq R$ 
be the Sylow r-subgroup of $T^{r^{2}}$. Since $T^{\pi^{2}}$ is nilpotent, $R$ is a direct factor of $T^{r^{2}}$ and $T^{r}$ centralizes no $G$-chief factor of $R / \Phi(R)$. But a $G$-chief factor of $R / \Phi(R)$ is a $T$-chief factor of $R / \Phi(R)$, and hence an $L$-composition factor of $R / \Phi(R)$. Thus Lemma 4 implies $l(R L)=l(R W D)=3$. Now $N R \unlhd G$, $C_{G}(N)=N$, and $N=$ Fit $(G)$. Thus $N$ is $R$-hypereccentric. Considering the group $N R W D$ we apply Lemma 4 and obtain $l(N R W D)=4$. The minimality of $G$ gives $G=N R W D$. Suppose that $R_{0}$ is a minimal normal subgroup of $R W D$ with $R_{0}<R$.

Suppose $N R_{0} W D=N R W D$. Then $R_{0} W D=T=R W D$ and $R=R_{0}(W \cap R)$. However $R / \Phi(R)$ is $\pi^{2}$-hypereccentric, so $W \cap R \leq \Phi(R)$. Consequently $R=R_{0} \Phi(R)$, contradicting $R_{0}<R$. Thus $N R_{0} W D<N R W D$. The minimality of $G$ implies $l\left(N R_{0} W D\right)=3\left(l\left(N R_{0} W D\right) \leq 2\right.$ implies $N \leq L=W D$, whereas $\left.N \cap W D \leq N \cap T=1\right)$. Then $\left(R_{0} W D\right)^{r^{2}}$ is trivial on each $N R_{0} W D$-chief factor contained in $N$. However $\left(R_{0} W D\right)^{r^{2}} \leq R_{0}$ and $\left(\left|R_{0}\right|,|N|\right)=1$. Thus $\left(R_{0} W D\right)^{r^{2}} \leq C(N) \cap R_{0}=N \cap R_{0}=1$, and $l\left(R_{0} W D\right)=2$. Now $R_{0}$ is minimal normal in $R_{0} W D$ and $R_{0} W D / C\left(R_{0}\right) \cap$ $R_{0} W D$ is nilpotent. Thus $W \leq C\left(R_{0}\right)$ and $D$ acts fixed-point-freely on $R_{0}$ (otherwise $R_{0} N / N$ would be a central factor in $G$ ). We are assuming that $C_{G}(D)$ is nilpotent. Since $N$ is the unique minimal normal subgroup of $G$ and since $G=N T$ with $N \cap T=1$, we have $N=C_{G}(N)$ and $(|N|,|R|)=1$. Our assumption that $C_{G}(D)$ is nilpotent is equivalent to $C_{R}(D) \leq C\left(C_{N}(D)\right)$.

Let $K$ be a finite splitting field for all subgroups of $G, \operatorname{char}(K)=q$. Then $K \otimes N=N_{1} \oplus \cdots \oplus N_{t}$, a direct sum of absolutely irreducible, algebraically conjugate representations of $R W D$. Also $R W D$ acts faithfully on each $N_{i}$. Now $C_{K \otimes N}(D)=K \otimes C_{N}(D)=C_{N_{1}}(D) \oplus \cdots \oplus C_{N_{t}}(D)$ and $C_{K \otimes N}\left(C_{R}(D)\right)=$ $K \otimes C_{N}\left(C_{R}(D)\right)=C_{N_{1}}\left(C_{R}(D)\right) \oplus \cdots \oplus C_{N_{t}}\left(C_{R}(D)\right)$. Thus letting $V=N_{1}$ we have $C_{R}(D) \leq C\left(C_{V}(D)\right)$. Apply Clifford's theorem to $R_{0}$. $V_{R_{0}}=V_{1} \oplus \cdots \oplus V_{n}$, a decomposition into homogeneous components. On each $V_{i}, R_{0}$ induces a group of scalar transformations. As $D$ is fixed-point-free on $R_{0}, D$ acts semiregularly on the $V_{i}$. Thus if $0 \neq x_{i} \in V_{i}$ then $x=\Sigma_{g} \in D_{i}^{x_{i}^{g}}$ is nonzero and centralized by $D$. Thus, $C_{R}(D)$ centralizes $x$. However $R_{0} \leq Z(R)$ implies that $R$ stabilizes each $V_{i}$ and so $C_{R}(D)$ stablizes each $V_{i}$. Consequently $C_{R}(D)$ centralizes $x_{i}$, and as $i$ and $x_{i} \in V_{i}$ were arbitrary, $C_{R}(D) \leq C(V)=1$. But this implies $D$ is fixed-pointfree on $R W$, whereas this group is not nilpotent. This is a contradiction and so $R$ is minimal normal in $R W D$.

Now let $Q$ be a minimal normal subgroup in $D W$. As $W$ is nilpotent, $D$ is fixed-point-free on $Q$. Consider the group $G_{0}=N R Q D$. Then the minimality of $G$ yields $G=G_{0}$ if $l\left(G_{0}\right)=4$. Suppose $l\left(G_{0}\right)=3$. Since $D$ is fixed-point-free on $Q$ and since $R$ acts without fixed points on $N$, the only possibility is that $Q \leq$ $C(R)$. Also $l(R W D)=3$ and $R$ minimal normal in $R W D$ implies that $R \cap W D=1$. 
As above write $K \otimes N=N_{1} \oplus \cdots \oplus N_{t}$, set $V=N_{1}$ and consider $V_{Q}=$ $V_{1} \oplus \cdots \oplus V_{b}$. D permutes the $V_{i}$ semiregularly and $R \leq C(Q)$ fixes each $V_{i}$. Once again we get the contradiction $C_{R}(D) \leq C(V)=1$. This completes the proof of the claim.

We now complete the proof of Theorem 5. We have $C_{R}(D) \leq C\left(C_{N}(D)\right)$. As above choose a finite field $K$ of characteristic $q$ such that $K$ is a splitting field for all subgroups of $G$, and obtain a faithful irreducible $K(R Q D)$-module $V$ satisfying $C_{R}(D) \leq C\left(C_{V}(D)\right)$. Write $V_{R}=V_{1} \oplus \cdots \oplus V_{k}$, where the $V_{i}$ are the homogeneous components. Then $Q D$ is transitive on $V_{1}, \cdots, V_{k}$. If $k=1$, then $R$ is cyclic, which is impossible since $Q D$ acts faithfully on $R$. Thus $k>1$.

If $D$ is semiregular on $V_{1}, \cdots, V_{k}$, then as in the above claim $C_{R}(D) \leq$ $C(V)=1$, a contradiction. Thus we may assume that $D$ fixes $V_{1}$. Since $C_{Q D}(D)$ is transitive on the $V_{i}$ 's fixed by $D, D$ fixes only $V_{1}$. Now $D$ is semiregular on $V_{2}, \cdots, V_{k}$ and it follows that $C_{R}(D) \leq C\left(V_{2} \oplus \cdots \oplus V_{k}\right)$. Thus $C_{R}(D)$ acts faithfully on $V_{1}$ and $C_{R}(D)$ is cyclic. Say $C_{R}(D)=\langle t\rangle$. The stabilizer of $V_{1}$ in $R Q D$ acts irreducibly on $V_{1}$, and this stabilizer is just $R D$. Now $R / \operatorname{ker} V_{1}$ is represented as scalar matrices and consequently $R / \operatorname{ker} V_{1}=C_{R}(D) \operatorname{ker} V_{1} / \operatorname{ker} V_{1}$. As $R D$ acts irreducibly and $R D /$ ker $V_{1}$ is abelian, we have $\operatorname{dim}\left(V_{1}\right)=1$. But then the matrix of $t$ has the form

$$
\left(\begin{array}{cccc}
a & & & \\
& 1 & & \\
& & & \\
& & \cdot & \\
& & & \\
& & &
\end{array}\right)
$$

and $\operatorname{det}(t)=\alpha \neq 1$. As $t \in R \leq(R Q D)^{\prime}$, this is impossible and the proof of Theorem 5 is complete.

Theorem 6. Let $n \geq 3$ and $G=A_{n} A_{n-1} \cdots A_{1}$ be a repeated semidirect product of the abelian groups $A_{i}$. Suppose that $A_{1}$ is a system normalizer of $G$, $A_{1}$ bas prime order $p$ and $\left(p,\left|A_{i}\right|\right)=1$ for $i>1$. If $l(G)=n$, then $l\left(C_{G}\left(A_{1}\right)\right)=$ $n-2$.

Proof. Let $G$ be a minimal counterexample. By Theorem $5 n>4$. Write $A_{1}=D$. If $\mathcal{T}=\pi^{n-1}$, then $1<G^{\mathcal{T}} \leq A_{n}$ and $G^{\mathcal{T}}$ is $\mathcal{T}$-hypereccentric [1, Theorem 5.15]. Let $N_{n}$ be a minimal normal subgroup of $G$ with $N_{n} \leq G^{\mathfrak{T}} . N_{n}$ is $\pi^{n-1}$. eccentric so that $G / C_{G}\left(N_{n}\right)$ has length $n-1$. As $A_{n} \leq C\left(N_{n}\right)$, setting $G_{0}=$ $N_{n} A_{n-1} \cdots A_{1}$ we have $N_{n}$ minimal normal in $G_{0}$ and $G_{0} / C_{G_{0}}\left(N_{n}\right)$ of length $n-1$. Thus $l\left(G_{0}\right)=n$ by Lemma 4 , and the minimality of $G$ implies that $G=G_{0}$. Also the minimality of $G$ implies that $C_{G}\left(N_{n}\right)=N_{n}$. Now let $N_{n-1}$ be a minimal 
normal subgroup of $A_{n-1} \cdots A_{1}$ contained in $\left(A_{n-1} \cdots A_{1}\right)^{r^{n-2}}$. Then the minimality of $G$ implies that $G=N_{n} N_{n-1} A_{n-2} \cdots A_{1}$ and $C_{G}\left(N_{n-1}\right)=N_{n-1}$. Continuing in this way we obtain $G=N_{n} \cdots N_{1}$ where for each $i=1, \cdots, n, N_{i}$ is self-centralizing and minimal normal in $N_{i} \cdots N_{1}$.

For each $i$, let $M_{i}=N_{i} \cap C(D)$. Then $M_{2}=1$. As $D=A_{1}$ is a Sylow $p$-subgroup of $G, C_{G}(D)=M_{n} \cdots M_{3} \times D$. We are assuming that $l\left(M_{n} \cdots M_{1}\right) \leq n-3$. Now $N_{n-1} \cdots N_{1}<G$, so that $l\left(M_{n-1} \cdots M_{1}\right)=n-3$. Thus $l\left(M_{n} \cdots M_{1}\right)=n-3$ and $M_{n}{ }_{n}$ is $\pi^{n-3}$-hypercentral. As ${ }^{n-1}\left(\left|M_{n}\right|,\left|M_{n-1}\right|\right)=1$ we have $\left(M_{n-1}^{n} \cdots M_{1}\right)^{\pi^{n-4}} \leq$ $C\left(M_{n}\right)$. Also $\left[M_{3}, M_{4}\right]=\left(M_{3} M_{4}\right)^{r},\left[M_{3}, M_{4}, M_{5}\right]=\left(M_{3} M_{4} M_{5}\right)^{r^{2}}, \ldots,\left[M_{3}, \ldots, M_{n-1}\right]$ $=\left(M_{n-1} \cdots M_{1}\right)^{r^{n-4}}$. Write $N_{3}=M_{3} \times L_{3}$ where $L_{3}=\left[D, N_{3}\right]$.

Suppose that $\left[\left[N_{4}, M_{3}\right], L_{3}\right] \neq 1$. Consider the group

$$
\begin{aligned}
G_{0}=D \cdot L_{3} \cdot\left[N_{4}, M_{3}, L_{3}\right]\left[N_{4}, M_{3}, L_{3}, N_{5}\right]\left[N_{4}, M_{3}, L_{3}, N_{5}, N_{6}\right] \\
\cdots\left[N_{4}, M_{3}, L_{3}, N_{5}, \ldots, N_{n}\right] .
\end{aligned}
$$

Then $G_{0}$ has the form $G_{0}=D L_{3} \cdots L_{n}$ where each $L_{i} \leq N_{i}$ and where $1<L_{i+1}=$ $\left[L_{i+1}, L_{i}\right]$, for $i=3, \cdots, n-1$. It follows that $D$ is a system normalizer of $G_{0}$ and $l\left(G_{0}\right)=n-1$. Thus $l\left(C_{G_{0}}(D)\right)=n-3$. $\left(C_{G_{0}}(D)\right)^{r^{n-4}} \leq C_{L_{n}}(D)$ so $C_{L_{n}}(D)$ is not $\pi^{n-5}$-hypereccentric. In particular $\left(C_{G_{0}}(D)\right) r^{n-5} \unrhd C\left(C_{L_{n}}(D)\right)$. That is $\left[C_{4}, C_{5}, \ldots, C_{n-1}\right] \not \subset C\left(C_{L_{n}}(D)\right)$, where $C_{i}=C_{L_{i}}(D)$. Now $C_{4} \leq\left[N_{4}, M_{3}, L_{3}\right] \leq$ $\left[N_{4}, M_{3}\right]$. As $M_{4}$ is abelian of order prime to $\left|M_{3}\right|, M_{3}$ centralizes no element of $\left[N_{4}, M_{3}\right]$. But $C_{4} \leq L_{4} \cap C(D) \leq N_{4} \cap C(D)=M_{4}$ and $M_{3}$ normalizes $M_{4}$. Thus $M_{4}=$ $\left[M_{4}, M_{3}\right] \times C_{M_{4}}\left(M_{3}\right)$ and $\left[M_{4}, M_{3}\right]=M_{4} \cap\left[N_{4}, M_{3}\right]$. It follows that $C_{4} \leq\left[M_{4}, M_{3}\right]$. Thus $\left[C_{4}, \cdots, C_{n-1}\right] \leq\left[M_{3}, M_{4}, \cdots, M_{n-1}\right]$, and so $\left[M_{3}, \ldots, M_{n-1}\right] \underline{\&}$ $C\left(C_{N_{n}}(D)\right) \leq C\left(C_{L_{n}}(D)\right)$. As $C\left(C_{N_{n}}(D)\right)=C\left(M_{n}\right)$, this is a contradiction. Thus $\left[N_{4}, M_{3}, L_{3}\right]=1$.

Consider the group $N_{4} N_{3} N_{2} D$. Write $N_{2}=S, N_{3}=Q, N_{4}=R, s, q$, and $r-$ groups, respectively. We have $Q=Q_{0} \times Q_{1}$ where $Q_{0}=C_{Q}(D)$ and $Q_{1}=[Q, D]$. Also $R$ is an irreducible $D S Q$-module satisfying $\left[\left[R, Q_{0}\right], Q_{1}\right]=1$. Let $K$ be a finite field of characteristic $r$ such that $K$ is a splitting field for each subgroup of $D S Q$. Then $K \otimes R=R_{1} \oplus \cdots \oplus R_{t}$ where the $R_{i}$ are absolutely irreducible, algebraically conjugate $D S Q$-modules. Since $\left[K \otimes R, Q_{0}\right]=K \otimes\left[R, Q_{0}\right]$ and $C_{K \otimes R}\left(Q_{1}\right)=K \otimes C_{R}\left(Q_{1}\right)$, it follows that $\left[R_{1}, Q_{0}, Q_{1}\right]=1$. Consider the representation of $D S Q$ on the module $V=R_{1}$. By Clifford's theorem $V_{Q}=$ $V_{1} \oplus \cdots \oplus V_{k}$ where the $V_{i}$ are the homogeneous components of $Q$ and the $V_{i}$ are permuted transitively by $D S$. If $k=1$, then $Q$ is cyclic and $D S Q / C(Q)=$ $D S Q / Q$ is cyclic. This is impossible, and so $k>1$. Let $V_{0}=\left[C_{V}(D), Q_{0}\right]$. By Theorem 5, $V_{0} \neq 0$ and since $V_{0} \leq\left[V, Q_{0}\right] \leq C_{V}\left(Q_{1}\right), V_{0}$ is $Q$-invariant. Thus $V_{0}=\left(V_{0} \cap V_{1}\right) \oplus \cdots \oplus\left(V_{0} \cap V_{k}\right)$. If $D$ is semiregular on $V_{1}, \cdots, V_{k}$ then 
$V_{0} \cap V_{i}=0$ for each $i$, contradicting the fact that $V_{0} \neq 0$. Thus $D$ is not semiregular, say $D$ fixes $V_{1}$. Then $C_{D S}(D)$ is transitive on the $V_{i}$ fixed by $D$, so that $D$ fixes just $V_{1}$. In particular $V_{0} \leq V_{1}$. The stabilizer of $V_{1}$ is $D Q$ and so $D Q$ acts irreducibly on $V$.

Let $0 \neq v_{i} \in V_{i}, i>1$. Then $v=\Sigma_{g \in D} v_{i}^{g}$ is nonzero and centralized by $D$. So for $t \in Q_{0}$, we have $v^{t}-v \in V_{0}$. But $v^{t}-v \in V_{2} \oplus \cdots \oplus V_{k}$, so that $v^{t}=v$ and $v_{i}^{t}=v_{i}$. It follows that $Q_{0}$ centralizes $V_{2} \oplus \cdots \oplus V_{k}$. Then $Q_{0}$ is faithful on $V_{1}$ and since $Q$ induces a cyclic group on $V_{1}$, we can write $Q_{0}=\langle t\rangle$. Then $D Q / \operatorname{ker}\left(V_{1}\right)=\left(D \times Q_{0}\right) \operatorname{ker} V_{1} / \operatorname{ker} V_{1}$ and $\operatorname{dim} V_{1}=1$. Then $t$ is represented on $V$ by the matrix

$$
\left(\begin{array}{llll}
a & & & \\
& 1 & & \\
& & & \\
& & \cdot & \\
& & & \\
& & & 1
\end{array}\right)
$$

for some $1 \neq \alpha \in K$. This contradicts the fact that $Q_{0} \leq Q \leq(D S Q)^{\prime}$, and Theorem 6 is proved.

One special case of Theorem 6 is a common one in the construction of solvable groups. Namely let $D=A_{1}$ have prime order $p$. Let $A_{2}$ be a finite faithful irreducible module for $D$ over a field of characteristic prime to $p$. Now let $A_{3}$ be a finite faithful irreducible module for the group $A_{2} A_{1}$ over a field of characteristic prime to $p$. Continue in this way and let $G=A_{n} \cdots A_{1}$. Then as long as $n \geq 3, l(G)=l(C(D))+2$.

The last result is another special case of Theorem 6 .

Theorem 7. Let $G$ be an A-group baving nilpotent length $n \geq 3$ and system normalizer $D$ of prime order. Then $l\left(C_{G}(D)\right)=n-2$.

Proof. Suppose $l(G)=n \geq 3, G$ is an $A$-group and $D$ is a system normalizer of prime order. $A_{n}=G^{\pi^{n-1}}$ is nilpotent and hence abelian. Thus $G=A_{n} T$ with $A_{n} \cap T=1$, for $T^{n}$ an $\pi^{n-1}$-normalizer of $G$ [1, Theorem 5.15]. Moreover we may assume $D \leq T$. Inductively we obtain the required factorization, $G=A_{n} \cdots A_{1}$. The theorem now follows from Theorem 6 .

We conclude the paper by giving examples that show two ways in which Theorem 6 is best possible.

Example 1. Let $A$ be cyclic of order $p^{3}$ and let $A$ act irreducibly on a group $B$ in such a way that $C_{A}(B)=\Omega_{2}(A)$. Now let $B A$ act irreducibly on a group $C$ so that $C_{B A}(C)=\Omega_{1}(A)$. Finally let $C B A$ act faithfully and irreducibly on a group $D$. Then $G=D C B A$ has length 4 , system normalizer $A$, and $l\left(C_{G}(A)\right)=1$. So the prime order assumption is needed. 
Example 2. Let $B A$ be the nonabelian group of order 21 , with $|B|=7,|A|=$ 3. Then $B A$ acts faithfully on an elementary group $C$ of order 8 , in such a way that $C_{C}(A)=\langle t\rangle$ where $t$ is an involution. Also $C=\langle t\rangle \times[C, A]$. Thus $C A$ has a linear representation $\Phi$ over $F_{3}$ with kernel $A[C, A]$. Let $V$ be the representation module of $\Phi^{C B A}$. Then $V$ is elementary abelian of order $3^{7}$ and $V$ is faithful. Let $G=V C B A$. Then $G$ satisfies the hypothesis of Theorem 5 and $l\left(C_{G}(A)\right)=2$.

Now the restriction of $V$ to $C$ splits as the direct sum of the seven distinct nontrivial linear representations of $C$ over $F_{3}$ and so $V=V_{0} \times V_{1}$ where $V_{0}=$ $[V, t], V_{1}=C_{V}(t),\left|V_{0}\right|=3^{4},\left|V_{1}\right|=3^{3}$. A centralizes $t$ and so acts on $V_{0}$. For $v \in V_{0},\left\langle v, v^{x}, v^{x^{2}}\right\rangle$ is $A$-invariant, where $\langle x\rangle=A$. Thus, under the action of $A$, $V_{0}$ splits as a sum of at least two indecomposable submodules, one of which has dimension 1 or 2 . This particular submodule contains an element $g$ such that $(x g)^{3}=1$ and $g \notin[V, A]$. Let $A^{\prime}=\langle x g\rangle$. Then $V C B A^{\prime}=G$ and $V C B \cap A^{\prime}=1$. However we will show that $C_{G^{\prime}}\left(A^{\prime}\right)$ is nilpotent. To see this first note that $C_{G}\left(A^{\prime}\right) V / V \leq C_{G}\left(A^{\prime} V / V\right)=C_{G}(A V / V)=A\langle t\rangle V / V$. Thus if $C_{G}\left(A^{\prime}\right) \& A V$, it must be that $A^{\prime}$ centralizes some element of $t V$. Suppose $x g$ centralizes $t v, v \in V$. Then $t v=(t v)^{x g}=\left(t v^{x}\right)^{g}=t^{8} v^{x}=t g^{2} v^{x}$. This gives $g=v^{-1} v^{x}=[v, x]$, a contradiction. This example shows that it is essential that the automorphism of prime order actually normalizes a Sylow system.

Finally, we remark that it may be possible to weaken the conditions on $A$, only assuming that $A$ is contained in a system normalizer of $G$. That is in Theorem 5 we would assume that $\left|G: G^{\pi}\right|=p,|A|=p$, and $A$ is contained in a system normalizer. This would allow for central factors contained in $G^{\pi}$.

\section{REFERENCES}

1. R. Carter and T. Hawkes, The I-normalizers of a finite solvable group, J. Algebra 5 (1967), 175-202. MR 34 \#5914.

2. G. Seitz and C. R. B. Wright, On complements of T-residuals in finite solvable groups, Arch. Math. (Basel) 21 (1970), 139-150. MR 42 \#6117.

3. J. G. Thompson, Fixed points of p-groups acting on p-groups, Math. Z. 86 (1964), 12-13. MR $29 \# 5911$.

DEPARTMENT OF MATHEMATICS, UNIVERSITY OF OREGON, EUGENE, OREGON 97403 